Polymer Journal, Vol. 39, No. 8, pp. 822-827 (2007)

(C) 2007 The Society of Polymer Science, Japan

\title{
One-Pot and Rapid Synthesis of Uniformed Silica Spheres via Mediation of Linear Poly(ethyleneimine)s and Dyes
}

\author{
Ren-Hua JIN ${ }^{\dagger}$ and Jian-Jun YUAN \\ Synthetic Chemistry Lab, Kawamura Institute of Chemical Research, 631 Sakado, Sakura 285-0078, Japan
}

(Received March 28, 2007; Accepted May 12, 2007; Published June 26, 2007)

\begin{abstract}
Fabrication of monodisperse silica spheres with bright emission features is still valuable in challenging since the spheres are widely applicable in the fields of catalysis, imaging material, bio-encapsulation, drug delivery, coatings, cosmetic, energy storage, and so on. In this paper, we demonstrated a new one-pot approach to uniformed silica spheres via mediation of linear poly(ethyleneimine)s (PEIs) and acidic dyes. PEIs with different architectures possessing only secondary amine in backbone were used as mediators for silica deposition from hydrolytic condensation of tetramethoxysilane (TMOS). Under the optimized medium composition of methanol/water with 70/30 in volume, the PEIs facilely promoted the hydrolytic condensation of TMOS to give rapidly and exclusively uniformed silica spheres. In this process, acidic dyes such as tetrakis(4-sulfonylphenyl)porphyrin (TSPP), tetrakis(4-carboxylphenyl)porphyrin (TCPP) can be incorporated with basic PEIs and play important role to produce relatively larger uniformed silica spheres entrapping the dyes inside. The diameters of the uniformed silica spheres ranged from 50 to $700 \mathrm{~nm}$ depending upon polymer architectures. From time-course investigation, it is confirmed that the formation of the silica spheres has three stages: 1) early formation of widely distributed smaller spheres; 2) growth to uniformed intermediate silica spheres and 3) further growth into larger spheres with narrow distribution. [doi:10.1295/polymj.PJ2006277]

KEY WORDS Uniformed Silica Spheres / Dye-embedded Silica Spheres / Biomimetic Silica / Linear Poly(ethyleneimine) / Porphyrins /
\end{abstract}

As we demonstrated in our recent work, polymers with linear poly(ethyleneimine) (LPEI) backbone are very effective mediators to deposit hierarchical silica with complex shapes and structures from hydrolytic condensation of alkoxysilane. ${ }^{1,2}$ For examples, the silicas from nanofiber-based web, film, plate, bundle, flower, star, dendrimer, fan, urchin and turbine-like shapes to nanosphere-based large aggregates are producible facilely by simply changing LPEI architectures and concentrations, by adjusting the media modulation and by incorporating with metal cations. Usually, LPEI easily forms fibrous-based aggregates due to its strong self-organization (crystalline) power in aqueous media and the fibrous-based aggregates act as reactors for silica deposition with transcribing ability. However, addition of methanol into the aqueous solution of LPEI can reduce the crystallization power of LPEI. For instance, high methanol content such as $50 \%$ in aqueous medium dramatically retarded the nucleation of the LPEI crystalline and thus the growth of the crystalline was not towards long filament but was suppressed within complex globular domains. ${ }^{1 \mathrm{c}, 2 \mathrm{~b}}$ These complex globular domains play as templates to direct the corresponding silica with remaining the shapes of the templates. ${ }^{2 b}$ Thus, media modulation with methanol provided reproducible feasibility to change the silica from nanofiber-based network to globular-based aggregates. ${ }^{1 \mathrm{c}-\mathrm{d}}$ Such significant shape changes were switched simply by changing the ratio of methanol to water in LPEI aggregation. This finding of the globular domain formation by methanol modulation promoted us to explore a route to uniformed silica sphere by mediation of LPEI and multi-site acidic molecular parts which can shrink the aggregates of LPEI via physical interactions between LPEI and the acids.

Recent progress in the so-called biomimetic synthesis of silica revealed that spherical silica is easily available under mild conditions with assisting natural and synthetic long chain polyamines. ${ }^{3}$ Silaffins extracted from diatom can be used as mediator to deposit spherical silica from silicic acid. ${ }^{4 a-d}$ Natural poly(propyleneimine)s with different chain length also effectively directed silica spheres. ${ }^{4 \mathrm{e}}$ Polypeptide with diblock architecture induced the hydrolytic condensation of TEOS resulting in silica spheres. ${ }^{5 a}$ Poly(allylamine) associated with phosphate was able to induce silica spheres. ${ }^{5 \mathrm{~b}-\mathrm{c}}$ Dendrimers with polyamidoamine and polypropyleneimine backbones serve as templates for directing silica spheres ${ }^{5 \mathrm{~d}-\mathrm{e}}$ Compared to the method of traditional Stöber silica spheres ${ }^{6}$ which requires great care during synthesis, all those polyamines mediated synthesis of spherical silica are attractive due to merits of one-pot and mild conditions. How-

${ }^{\dagger}$ To whom correspondence should be addressed (E-mail: jin@kicr.or.jp). 


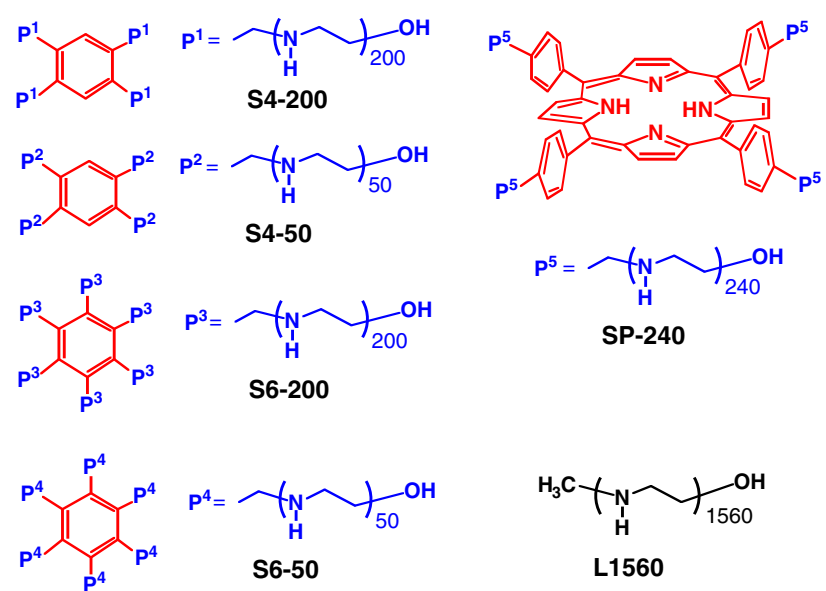

Scheme 1. Polymers with linear poly(ethyleneimine) backbone.

ever, synthesis of definite monodisperse silica spheres with functionalized feature is still challenging by those so-called polyamines mediation.

As a systematic expanding of our silica architectures, herein, we describe a very simple LPEI based one-pot manner to fabricate uniformed silica spheres with entrapping acidic dyes. Under an optimized medium composition of methanol/water at 70/30 in volume, the PEIs with linear backbone but different architectures directed monodisperse silica spheres (ranged form 50 to $700 \mathrm{~nm}$ in diameters) with functional parts inside only within $1 \mathrm{~h}$ at room temperature.

\section{EXPERIMENTAL}

\section{Materials}

Poly(ethyleneimine)s such as S4-200, S4-50, S6200, S6-50, SP-240 and L1560 shown in Scheme 1 were synthesized according to the previously reported methods (the number behind hyphen means averaged polymerization degree of the arms, details see supporting information). Tetramethoxysilane (TMOS) and tetrakis(4-sulfonylphenyl)porphyrin (TSPP), tetrakis(4-carboxylphenyl)porphyrin (TCPP) were purchased from Aldrich.

\section{Characterization}

The silica morphologies were viewed by scanning electron microscope (SEM) with Keyence VE7800 microscope. TEM and HR-TEM images were obtained by JEM-2200FS (JEOL) transmission electron microscopes operated at $200 \mathrm{kV}$. The fluorescence images of porphyrin-encapsulated silica spheres were visualized with a video enhanced optical microscope (Olympus BX-60 equipped with a CB-300Z/OL digital camera). The electrospectroscopic features of porphyrin residues in solution and in silica spheres were characterized with Hitachi U-3500 UV-vis spectrometer and Hitachi F4500 fluorescence spectrometer. The UV-vis spectra were recorded with an integration spherical attachment. The spheres distributions were evaluated by Macview Particle Analyze System (Mountech) via counting the spheres seen in SEM images.

\section{Preparation of LPEIs Solution and Silica Deposition}

Polymers with LPEI backbone were firstly dissolved in methanol in $1 \%$ concentration with or without TSPP (molar ratio of $-\mathrm{CH}_{2} \mathrm{CH}_{2} \mathrm{NH}-/ \mathrm{TSPP}=$ 1200/1). Then, a stock polymer solution was prepared by mixing the methanol solution with water by $7 / 3$ volume ratios. To the stock polymer solution $(1 \mathrm{~mL}$, $\mathrm{pH}=9.2$ ) was added a desired amount of TMOS or TMOS/ethanol solution (see text) under shaking and then left for $40 \mathrm{~min}$ at room temperature. The silica particles obtained were washed by centrifugation with ethanol three times and dispersed in ethanol.

\section{RESULTS AND DISCUSSION}

Linear poly(ethyleneimine) is easily soluble in methanol at ambient conditions although it is not soluble in water at room temperature. In our previous study of LPEI mediated silica synthesis, we confirmed that pre-organized LPEI aggregates in aqueous or water/methanol media just serve templates in which TMOS added is rapidly hydrolyzed around the LPEI aggregates to give shaped silica with fibril-, fun- and flower-like morphologies. ${ }^{1 \mathrm{a}, 2 \mathrm{~b}}$ In contrast, addition of TMOS to the branched PEI solution without pre-organization only produced bulky silica. ${ }^{2 a}$ Also, LPEI system with excess of methanol afforded bulky silica without shape control. ${ }^{1 \mathrm{c}-\mathrm{d}}$ That is the pre-organized LPEI aggregate is an indispensable condition for producing the shaped (non-spherical) silica. However, in the media of $\mathrm{MeOH} /$ water $=70 / 30(\mathrm{vol} / \mathrm{vol})$, polymers with LPEI backbone such as S4-200, S4-50, S6-200, S6-50, SP-240 and L1560 (Scheme 1) or mixture of the polymers and TSPP were always transparent and we could not find any pre-organized aggregates even by means of dynamic light scattering (DLS) and microscopes. This means that the media $\mathrm{MeOH} /$ water $=70 / 30(\mathrm{vol} / \mathrm{vol})$ did not allow the pre-organization of LPEI.

With this in mind, we performed the following silicification experiments by alternative addition of water into reaction mixtures. In one experiment, to a mixture including $1.0 \mathrm{~mL}$ of tetramethoxysilane (TMOS) and $0.7 \mathrm{~mL}$ of methanol solution of S4200/TSPP (1\% of S4-200, [EI]/[TSPP] = 1200/1) was added water $(0.3 \mathrm{~mL})$ to induce silicification. In another one, to a mixture containing $0.7 \mathrm{~mL}$ of meth- 

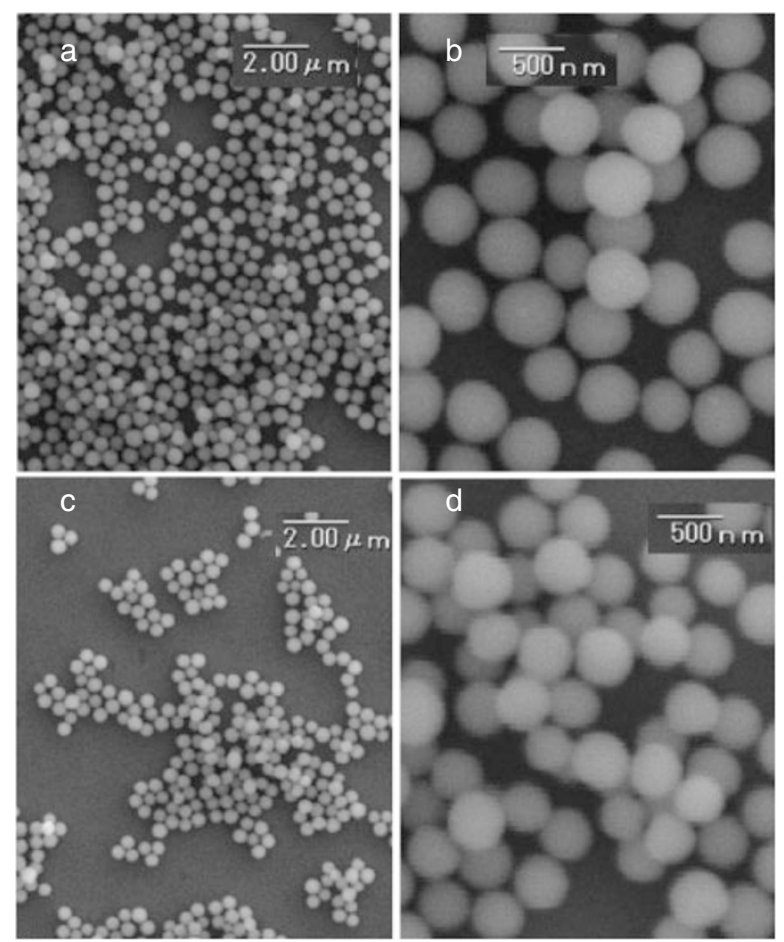

Figure 1. SEM images of the silica spheres mediated by S4$50 /$ TSPP by changing the addition sequence of water and TMOS. ( $a$ and $b$ ) water added behind TMOS; (c and d) water added ahead of TMOS. ( $b$ and $d$ ) magnified images of ( $a$ and $c$ ).

anol solution of S4-200/TSPP (1\% of S4-200, [EI]/ $[$ TSPP $]=1200 / 1)$ and $0.3 \mathrm{~mL}$ of water was added TMOS $(1.0 \mathrm{~mL})$ immediately. The two final mixtures were placed at room temperature for $40 \mathrm{~min}$. SEM images (Figure 1) of the silica particles resulted from the two cases indicate that both cases afforded the same size silica spheres with near $400 \mathrm{~nm}$ in diameter. Considering the fact that the hydrolytic condensation of TMOS occurs suddenly in aqueous solution of LPEI, we expect that the silicic acid produced in-situ from hydrolysis of TMOS in the presence of water would interact with LPEI/TSPP to form subtle aggregates which promote the nucleation and the growth of the uniformed spherical silica. In order to investigate the effect of TSPP in silicification, we prepared two kinds of $1 \%$ polymers solutions in methanol such as containing polymer only and polymer incorporated with TSPP, and used them in silica deposition from TMOS. As a typical example, to $0.7 \mathrm{~mL}$ of methanol solution of S4-200 ( $1 \mathrm{wt} \%, 1200$ eq monomer unit) containing TSPP ( 1 eq) was added $0.3 \mathrm{~mL}$ of water and then the solution was mixed with a solution containing $1 \mathrm{~mL}$ of TMOS and $0.5 \mathrm{~mL}$ of ethanol under stirring and the mixture was placed statically for $40 \mathrm{~min}$ at room temperature. On the other hand, the same reaction but without TSPP was also performed. The particles produced from the two reactions were washed with ethanol by centrifugation and subjected to SEM and
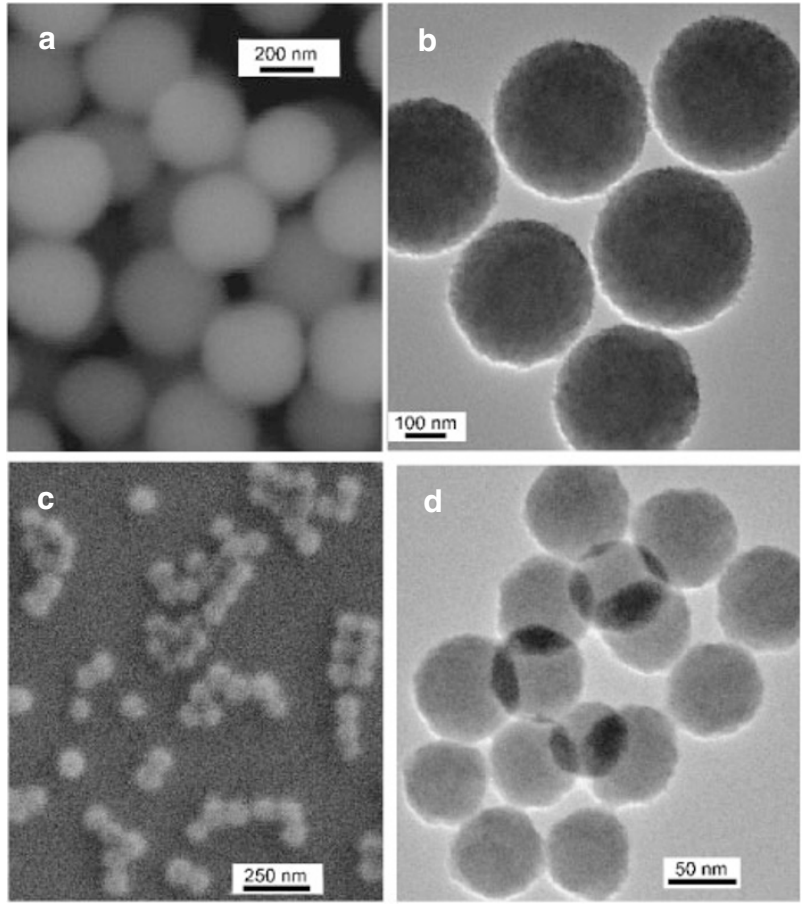

Figure 2. SEM (a and c) and TEM (b and d) images of the silica spheres mediated from S4-200/TSPP (a and b) and S4200 (c and d).

TEM observation. Figure 2 showed SEM and TEM images of the resulting silica particles. Evidently, the silica particles are uniformed spheres but the diameter (300 nm in TEM) mediated from S4-200/ TSPP is nearly 6 times larger than other one $(50 \mathrm{~nm}$ in TEM) mediated from S4-200 solution without TSPP (Figure $2 b$ and $d$ ). From the highly magnified TEM image (Figure S1a), we clearly observed that many grains sized several nanometers exist in the surface of the larger sphere. In comparison, the silica particles without TSPP did not have nano grains in the surface but seem rough (Figure S1b). In addition, thin-sectioned slices (Figure S1c) from the larger spheres with TSPP were visualized by TEM and no any cavity inside was found. Considerably, TSPP plays an important role in forming relatively larger silica spheres with dense silica skeleton although only a trace of TSPP was incorporated with S4-200 for silica deposition. Replacing TSPP with tetrakis(4carboxylphenyl)porphyrin (TCPP), we also obtained larger spherical silica (Figure S2). It needs to note that change the ratio of $[\mathrm{EI}] /[\mathrm{Dye}]$ with increasing porphyrin quantity (such as $[\mathrm{EI}] /[\mathrm{Dye}]=500 / 1$ ) is not good for preparing uniformed spherical silica. The reason is mainly due to the low solubility of the porphyrin dyes in the mixed water/MeOH solution.

It is notable that once TMOS was added to the aqueous solution of LPEI/TSPP, the formation of silica spheres began. This is very rapid. In order to reveal dynamic process of the formation of the spherical sili- 

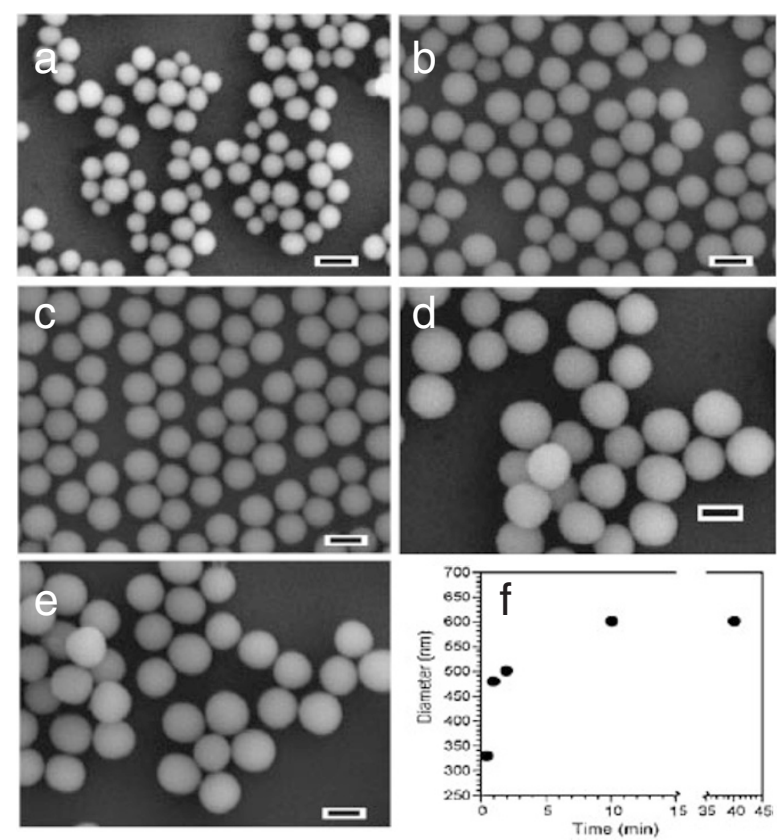

Figure 3. SEM images of the silica spheres formed at different reaction time. (a) $330 \pm 42 \mathrm{~nm}, 0.5 \mathrm{~min}$; (b) $475 \pm 45 \mathrm{~nm}, 1.0$ $\min$; (c) $500 \pm 43 \mathrm{~nm}, 2 \mathrm{~min}$; (d) $600 \pm 50 \mathrm{~nm}, 10 \mathrm{~min}$; (e) $600 \pm$ $40 \mathrm{~nm}, 40 \mathrm{~min}$; (f) the plot of mean diameters $v s$ reaction time.

ca, we investigated the time dependence of the spherical silica formation employing the star S4-50. To $0.7 \mathrm{~mL}$ methanol solution of S4-50/TSPP (1200/1 molar ratio), $0.3 \mathrm{~mL}$ water was added and the mixture was placed overnight. To the mixture was added a solution containing TMOS $(1.2 \mathrm{~mL})$ and EtOH $(0.3 \mathrm{~mL}$, use of small amount of EtOH is helpful to rapidly dissolve TMOS in the resulting reaction mixture). A trace of sample taken out from the reaction mixture at different time was cast on a slide glass and subject to SEM observation. Figure 3 showed the SEM images of the silica particles obtained from the timecourse. At early $30 \mathrm{~s}$, the reaction mixture gave widely distributed silica spheres ranged from 200 to $400 \mathrm{~nm}$ in diameters. The spheres obtained by 1 and $2 \mathrm{~min}$ were uniformed to near $500 \mathrm{~nm}$ in diameters. As the reaction time further elongated to 10 and $40 \mathrm{~min}$, the corresponding silica spheres were ranged about 600 $\mathrm{nm}$ in diameters. It seems that the formation of the silica spheres has three stages: 1) early formation of widely distributed smaller spheres; 2) growth to uniformed intermediate silica spheres and 3) further growth into larger spheres with narrow distribution. In addition, it is notable that the silica spheres mediated from S4-50/TSPP are apparently larger than that from S4-200/TSPP.

The polymers with different architecture such as L1560, S6-200, S6-50 and PS-240 also directed uniformed silica spheres in the presence of TSPP as long as the medium of methanol/water remained at the ra-
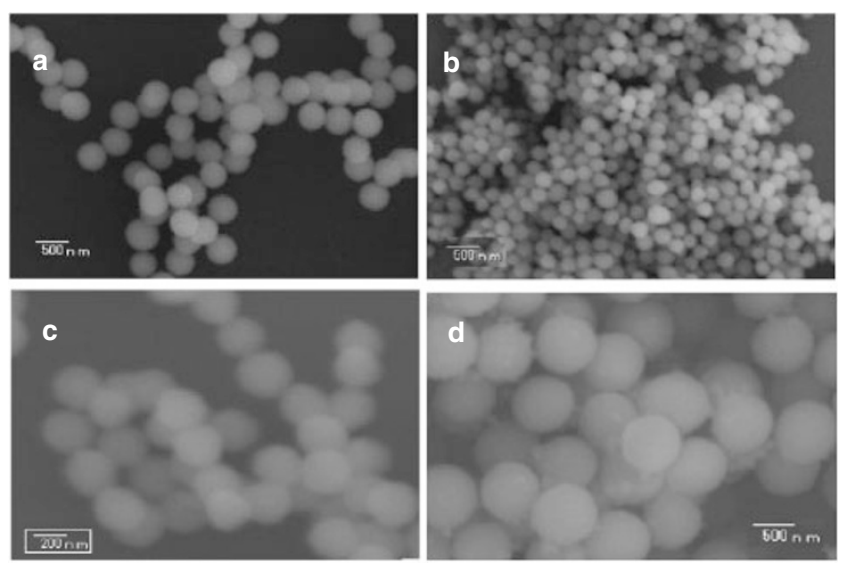

Figure 4. SEM images of the silica spheres mediated by different polymers: (a) $250 \pm 22 \mathrm{~nm}$ for S6-200; (b) $300 \pm 25 \mathrm{~nm}$ for SP-240; (c) $450 \pm 26 \mathrm{~nm}$ for L1560 and (d) $750 \pm 32 \mathrm{~nm}$ for S6-50.

tio $\mathrm{MeOH} / \mathrm{H}_{2} \mathrm{O}=7 / 3$ in volume. However, the diameters of the spheres changed with the PEI architectures (see Figure 4). It is clear that the star S6-200 with high polymerization degree tends to give smaller silica spheres than the S6-50. This tendency also appeared in the systems using S4-200 and S4-50 (see mentioned above). Therefore, it is suggestive that in the presence of TSPP, the stars with the longer arms favor to give the smaller silica spheres while the stars with the shorter arms do the larger ones. In the silica sphere formation, however, the volume ratio of methanol to water is most important factor to determine whether uniformed silica spheres forms. When the ratio of methanol/water was 50/50, the resulting silicas were flower-like but not uniformed spheres even in the presence or absence of TSPP. ${ }^{\text {b }}$

The attractive feature in our approach is that encapsulation of photofunctional dyes into the resulting silica spheres is easy and simple. Figure 5 showed light and fluorescence microscope images of the silica spheres mediated by S4-200/TSPP. The red fluorescence image (Figure 5b) indicates that the porphyrin dye incorporated into the silica spheres is sensitively photo responsible. We measured the absorption and fluorescence spectra of the uniformed silica spheres dispersed in methanol. It is observed that the absorption lines of the silica spheres mediated by S4-200/ TSPP (1200/1 in molar ratio) showed the same peak position with the methanol solution of S4-200/TSPP (1200/1 in molar ratio) (Figure S3). This result strongly indicates that the porphyrin residues entrapped in the silica spheres exist as molecularly distributed (i.e., isolated without stacking) state. In contrast to the absorption spectra in which absorption intensities of both the spheres and S4-200/TSPP solution increased with increasing concentrations, the emissions of both the spheres and S4-200/TSPP solution showed differ- 

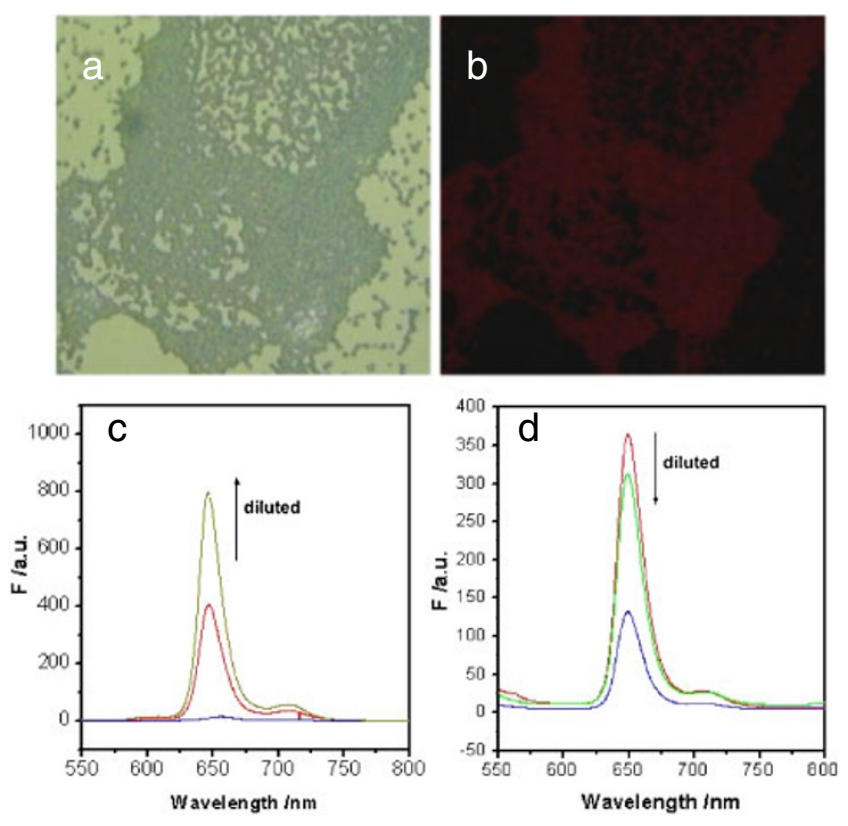

Figure 5. Optical (a) and fluorescent (b) microscopic images of the silica spheres mediated by S4-50/TSPP (1200/1 in molar ratio). Fluorescence spectra of S4-50/TSPP solution (c) and TSPP-entrapped silica spheres (d) with diluting the concentrations.

ent feature upon concentrations. The emission intensity (at $650 \mathrm{~nm}$ ) of the original methanol solution S4200/TSPP is very weak compared to those solutions diluted due to dynamic-induced self-quenching of TSPP at higher concentration. It is suggestive that in the S4-200/TSPP methanol solution system, the porphyrin residues are not tethered to the star polymer and thus are in moving with collision. After silica deposition from the solution medium, however, the supernatant did not show apparent fluorescence and absorption signals in its spectra while silica particles showed pale red. The emission intensity of the resulting silica spheres in methanol increased at higher concentration. Therefore, it is conclusive that TSPP residues with S4200 were almost completely encapsulated in the resulting silica spheres and existed inside as isolation status. We observed that the dye enclosed silica spheres were not bleached even dispersed in methanol over 1 year. Based on this feature, we can say that our approach via LPEI mediation to the silica spheres with dye encapsulation would be superior to the many improved Stöber routes in which the dyes incorporation into the Stöber silica spheres is not easily controllable because there are some procedures of chemically bonding dyes onto silica source or of physically doping dyes onto silica skeleton. ${ }^{7}$

In the biomimetic silica synthesis, phosphate was often used with association of polyamines for directing silica spheres. Morse et al. denoted that the phosphate plays as cross-linker between polyamines to form micellar domain which serves a template for spherical silica deposition. ${ }^{5 \mathrm{c}}$ Instead of TSPP, we used $\mathrm{H}_{3} \mathrm{PO}_{4}$ for silica deposition in $\mathrm{MeOH} / \mathrm{H}_{2} \mathrm{O}(70 / 30)$ medium by changing the molar ratio of S4-200 (EI unit) $/ \mathrm{H}_{3} \mathrm{PO}_{4}$ from $10,15,150$ to 1200 . When the ratio was 10 (i.e., $\mathrm{H}_{3} \mathrm{PO}_{4}$ concentration was relatively high) the system did not deposit silica at all; as the ratio ranged to 150 and 1200 fiber-based silica with complex shapes was resulted. Only the case with the ratio at 15, irregular silica particles ranged $40-150 \mathrm{~nm}$ were afforded effectively (see Figure S4). But, this result is not to be compared with the case of only using S4200. It seems that in our LPEI system the inorganic acid such as $\mathrm{H}_{3} \mathrm{PO}_{4}$ is not a candidate to produce uniformed silica spheres.

Although the mechanism of the sphere formation in LPEI system is not clear at present, we assume the procedure of forming the spherical silica as follows. In general, linear poly(ethyleneimine) backbone possessing only secondary amine residues has specific all trans zig-zag conformation in aqueous media. However, excess of methanol will suppress the extension tendency of LPEI. When TMOS is added, the LPEI rapidly hydrolyzes it into $\mathrm{Si}(\mathrm{OH})_{4}$ that are acidic species. The acid $\mathrm{Si}(\mathrm{OH})_{4}$ formed in-situ interacts with basic LPEI to induce micelle-like globular clusters. These clusters promote rapidly the nucleation from which the growth proceeds to form spherical intermediate domain and the polycondensation of the residual silicic acid continues around the domain until the LPEI part is totally covered by silica. Consequently, the outer surface of the final silica spheres is pure silica layer which terminates the sphere growth due to the absence of the catalyst of LPEI on this layer. In case of the presence of trace amount of TSPP, the TSPP might play as a special physical cross-linker causing loose association between the micelle-like clusters directing to relatively larger silica spheres. Here, it should be noted that no polymer core existed in the resulting spheres, which is supported by the TEM images of the sectioned slices of the silica spheres (see Figure S1c).

\section{CONCLUSIONS}

As conclusions, we successfully demonstrated a novel method to fabricate the uniformed silica spheres via LPEI mediation with additional acidic dyes. In this method, the uniformed and fluorescent silica spheres is available by one-pot within short time from hydrolytic condensation of alkoxysilane. Although some questions such as nucleation mechanism, the role of TSPP in enlarging sphere size need further investigation, our simplified method described here would provide a new insight and interest for materials scien- 
tists to tailor-made and develop monodisperse silica spheres, containing organic functional acidic parts inside, that are applicable as imaging, optical, photonic, medical and cosmetic materials.

\section{Electronic Supporting Information Available:} Figures S1, S2, S3, and S4. These materials are available via. the Internet at http://www.spsj.or.jp/c5/pj/ pj.htm

\section{REFERENCES}

1. a) R.-H. Jin and J.-J. Yuan, Chem. Commun., 1399 (2005). b) J.-J. Yuan and R.-H. Jin, Adv. Mater., 17, 885 (2005). c) J.-J. Yuan and R.-H. Jin, Langmuir, 21, 3136 (2005). d) R.-H. Jin and J.-J. Yuan, Macromol. Chem. Phys., 206, 2160 (2005).

e) P.-X. Zhu, N. Fukazawa, and R.-H. Jin, Small, 3, 394 (2007).

2. a) J.-J. Yuan, P.-X. Zhu, N. Fukazawa, and R.-H. Jin, $A d v$. Fucnt. Mater., 16, 2205 (2006).

b) R.-H. Jin and J.-J. Yuan, Chem. Mater., 18, 3390 (2006).

3. a) D. E. Morse, Trends Biotechnol., 17, 230 (1999).

b) C. W. P. Foo, J. Huang, and D. L. Kaplan, Trends Biotechnol., 22, 577 (2004).

c) M. Sumper and N. Kroger, J. Mater. Chem., 14, 2059 (2004).

d) S. V. Patwardhan, S. J. Clarson, and C. C. Perry, Chem Commun., 1113 (2005).
4. a) N. Poulsen, M. Sumper, and N. Kröger, Proc. Natl. Acad. Sci. U.S.A., 100, 12075 (2003).

b) N. Kröger, S. Lorenz, E. Brunner, and M. Sumper, Science, 298, 584 (2002).

c) N. Kröger, R. Deutzmann, and M. Sumper, Science, 286, 1129 (1999).

d) M. R. Knechi and D. W. Wright, Chem. Commun., 3038 (2003).

e) N. Kröger, R. Deutzmann, C. Bergsdorf, and M. Sumper, Proc. Natl. Acad. Sci. U.S.A., 97, 14133 (2002).

5. a) J. N. Cha, G. D. Stucky, D. E. Morse, and T. J. Deming, Nature, 403, 289 (2000).

b) M. Sumper, Angew. Chem., Int. Ed., 43, 2251 (2004).

c) E. Brunner, K. Lutz, and M. Sumper, Phys. Chem. Chem. Phys., 6, 854 (2004).

d) M. R. Knecht and D. W. Wright, Langmuir, 20, 4728 (2004).

e) M. R. Knecht, S. L. Sewell, and D. W. Wright, Langmuir, 21, 2058 (2005).

6. W. Stöber, A. Fink, and E. Bohn, J. Colloid Interface Sci., 26, 62 (1968).

7. a) L. M. Rossi, L. Shi, F. H. Quina, and Z. Rosenzweig, Langmuir, 21, 4277 (2005).

b) A. P. R. Johnston, B. J. Battersby, G. A. Lawrie, and M. Trau, Chem. Commun., 848 (2005).

c) H. Ow, D. R. Larson, M. Srivastava, B. A. Baird, W. W. Webb, and U. Wiesner, Nano Lett., 5, 113 (2005). d) Y. Chan, J. P. Zimmer, M. Stroh, J. S. Steckel, R. K. Jain, and M. G. Bawendi, Adv. Mater., 16, 2092 (2004). 